\title{
Locating distribution/service centers based on multi objective decision making using set covering and proximity to stock market
}

\author{
Mazyar Dabibi ${ }^{\mathrm{a}}$, Babak Farhang Moghaddam ${ }^{\mathrm{b}^{*}}$ and Mohammad Ali Afshar Kazemi ${ }^{\mathrm{c}}$
}

${ }^{a}$ Department of Management (MBA) ,Electronic Branch, Islamic Azad University, Tehran, Iran

${ }^{b}$ Institute for Management and Planning Studies, Tehran, Iran

${ }^{c}$ Department of Industrial Management, Electronic Branch, Islamic Azad University, Tehran, Iran

\section{CH R O N I C L E A B S T RA C T}

Article history:

Received November 42015

Received in Revised Format

December 212015

Accepted March 82016

Available online

March 92016

Keywords:

Marketing mix

Set covering problem

GA

Customer satisfaction

Facility location

Multi objective Optimization

\begin{abstract}
In the present competitive world, facility location is an important aspect of the supply chain (sc) optimization. It involves selecting specific locations for facility construction and allocation of the distribution channel among different SC levels. In fact, it is a strategic issue which directly affects many operational/tactical decisions. Besides the accessibility, which results in customer satisfaction, the present paper optimizes the establishment costs of a number of distribution channels by considering their proximity to the stock market of the goods they distribute, and proposes mathematical models for two objective functions using the set covering problem. Then, two objective functions are proposed into one through the $\varepsilon$-constraint method and solved by the metaheuristic Genetic Algorithm (GA). To test the resulted model, a smaller scale problem is solved. Results from running the algorithm with different $\varepsilon$-values show that, on average, a $10 \%$ increase in $\varepsilon$, which increases the value of the second objective function - distance covered by customers will cause a $2 \%$ decrease in the value of the first objective function including the costs of establishing distribution centers). The repeatability and solution convergence of the twoobjective model presented by the GA are other results obtained in this study.
\end{abstract}

(C) 2016 Growing Science Ltd. All rights reserved

\section{Introduction}

Nowadays, marketing relies not only on the production facilities, but only on the customer satisfaction. This approach brings up new market-related concepts and changes the principles of selling goods and services. Therefore, companies try to make a difference through providing their best services for their customers and compete with their rivals in such challenging areas; "proximity to customer" is a service companies provide to take over their rivals. If a company can provide its customers with appropriate services, there will be more customer satisfaction, sale increase, and profitability.

When there are limited resources and facilities as well as the necessity to meet their customers' needs, companies should first identify and prioritize the elements that affect customer satisfaction and then take necessary measures to enhance it accordingly. Customer satisfaction is something beyond a mere side-

* Corresponding author.

E-mail: farhang@iust.ac.ir (B. Farhang Moghaddam)

(C) 2016 Growing Science Ltd. All rights reserved.

doi: 10.5267/j.ijiec.2016.3.002 
effect of a company's efforts; it does not merely cause the personnel to work effectively and productively, it is the company's source of profit; customers are the reasons for a company to survive. When customers are satisfied with the provided services, their attitude is improved towards the company and this will enhance their satisfaction (Anderson \& Lindestad, 1998). Kotler believes that satisfaction is a feeling of happiness/despair caused by a comparison between a person's perceptions and expectations (Santouridis \& Trivellas, 2010).

Marketing Mix is a fundamental concept in the modern marketing. These elements, that should be capable of affecting the demand, make a set of controllable marketing variables the company mixes in its objective market to create the required reaction. Marketing mix is a set of controllable marketing tools including product, price, place/distribution, and promotion to mix in order to meet the requirements of the objective market. One of the marketing mix elements, which plays essential role for the producers' success in the industrial markets is associated with the company's distribution system. In fact, when we consider the globalization phenomenon and the increase competition in industries, the need for an integrated and coordinated distribution system is felt more than ever before. Location means all the activities carried out to deliver the product to or provide service for the customers and include distribution channels, commodity matching, distribution points, inventory level, and transport. Location models have broad nature and scope and each one looks for an optimum facility location through considering specific conditions. Many of the operational/tactical decisions are directly influenced by facility location. The main objective in facility location problems is to find the optimum location so as to minimize the total costs. 1909 is known by many people as the year when facility location theory was born (Ye, 2011); Alfred Weber was a theoretician who first proposed theories regarding facility location and cost minimization in that year and many other related theories and models were presented (Klose, 2005). Since 1960, the location theory attracted much attention; Brandeau and Chiu (1989) introduced 50 kinds of location problems and studied their interrelations. In a case study, Farahani and Asgari (2007) used multi-objective decision making models and investigated the number of warehouses required in a military logistics system (as distribution centers) by considering the minimum number and the best possible locations. Schmidt and Doerner (2010) presented a multi-period model for the location of emergency centers wherein the time spent by the ambulance was different for different areas; the ambulance location was so determined in their model as to definitely support the standard coverage from an area. In another case study related to the transportation industry, Malekinezhad et al. (2011) proposed a multi-objective set covering technique wherein different issues were studied by an integrated model under two topics of number and quality for which they utilized two different analytical methods. Chiu et al. (2011) studied the location of the waste recycling centers in Taiwan using the set covering method. Wang et al. (2011) proposed a decision making model for the location of the production and distribution centers and the inventory costs to reach some specific level of integration in the entire supply chain. Hosseininezhad et al. (2013) developed a location model of separate covering in a continuous space wherein the objective results include risk/installation costs, and uncertainties about customers' covering radius and installation costs allocated to an area with a predetermined covering radius are added to the objective function; the best place for facility installation is determined after the model is solved. Dell'Olmo et al. (2014) developed a maximal covering location model to determine the optimum points to install surveillance cameras in urban traffic network crossovers. To hierarchically solve the maximal covering location problem, Farahani et al. (2014) combined the covering and hierarchical models and developed an algorithm that uses the common solutions based on their compatibility to produce a new model that helps improving their efficiency. Dinler et al. (2014) proposed some mathematical programming formulae for single-/multi-objective facility location problems and solved and compared them through different heuristic methods. Akgun et al. (2015) proposed the pre-disaster facility location so as to minimize the risk that some demand points may not be covered by the located facilities. Arjmand et al. (2015) presented a novel mathematical model for facility location, routing, and available lands, and solved it by the genetic algorithm. They used GA to solve a new two-objective model for the location and transportation of hazardous materials (Arjmand et al., 2016). 
In the modern management, decision making is known to be the managers' most important task and responsibility, and the failures many people or agencies experience during their life-time highly depend on their decision making under critical conditions. In the management science, the decision theory presents a systematic and analytical method in decision making studies. An appropriate decision is one made based on logic; that is, it selects by necessity after it first identifies all the problem data and governing choices. Management of today's organizations and, hence, making decisions at this level is done under different economical, social, political, and in general, various environmental conditions; this means that decision making necessitates the consideration of numerous criteria (Tang et al., 2013). Multiobjective decision making evaluates decision choices based on various and, often, contradictory criteria. Model, a pattern adopted from reality, shows the relations between variables and can be utilized for decision making predictions. Using a model, we can make the most desirable decision without the risk of the real world decision making. The multi-criteria decision making models are divided into two major groups of Multiple Objective Decision Making (MODM) and Multiple Attribute Decision Making (MADM). In the first, several objectives are optimized simultaneously, but the evaluation scale for an objective might be different from those of the others, and in the second, one choice is selected from among the other existing ones.

There are different methods for the solution of multi-objective optimization problems one of which is $\varepsilon^{-}$ constraint (first proposed by Haimes et al., 1971). It is based on converting a multi-objective optimization problem to a single-objective one. In this method, one of the objective functions is selected and the rest are considered as constraints within some predetermined limits. Metaheuristic evolutionary methods too can be used for the solution of optimization problems. A nature-based optimization algorithm can effectively (and, most probably, quite successfully) search very large spaces in relatively short time intervals with the objective of finding global optimum solutions. Genetic algorithm (GA), an evolutionary optimization/search algorithm, is based on the principles of the genetic science and natural selection. In GA, a group of strings is created and grown with the general objective of maximizing the fitness of the whole population or minimizing the population-related costs. Holland, the first who did valuable works in this regard in the $60 \mathrm{~s}$ and $70 \mathrm{~s}$, made this algorithm popular (Aickelin, 2002), but, finally, Goldberg, one of his students, collected them (Wang et al., 2014). Holland's earlier works had been already compiled, printed, and published in his book and he was the first who tried to provide a theoretical foundation for genetic algorithms through stating the theory of patterns. Hosage and Goodchild (1986) were the first to use this algorithm on location-allocation problems. Jaramillo et al. (2002) studied the genetic performance on different location problems and compared the results. Ren and Awasthi (2015) investigated four metaheuristic methods (GA, Simulated Annealing, Taboo Search, and Ants Colony) for the solution of capacitated location-allocation problems in the logistic network.

Presence of a great number of consumers/customers to buy the desired products in the related stock market will result in the reduced marketing costs; therefore, determination of appropriate distribution centers that consider the distance to the rivals, regional covering, and optimized establishment costs (for the investors) is of utmost importance. Since appropriate locations will attract many customers, the researchers' objectives have been to develop a mathematical model and its related solution algorithm to find appropriate locations for the distribution of goods or services so as to reduce the company's costs as well as consider its presence in the stock market centers and covered areas.

\section{Research methodology}

Set Covering Problem, an old location problem, looks for a way to cover a subsystem of customers considering one or more objectives. Set covering is an optimization method that models many source selection problems. The objective is to select appropriate distribution/service centers to minimize the costs of meeting the demands to achieve a certain level of covering. In a covering problem, the demand is assumed covered when the facility is located in the radius of coverage. In the LSCP (Location Set Covering Problem) the objective is to find an appropriate facility location to minimize the cost to achieve a certain level of coverage. To make the set covering problem clearer, consider Fig. 1 where the sets of 
customers' locations are shown with solid circles. Suppose big circles are the geometrical models of facility/distribution centers' coverage areas. The objective will be to find the best location to place goods/services distribution centers so as to provide service for all customers with minimum number of the centers; ideally, every center is to cover the maximum number of customers. In Fig. 1, there are 22 points to be covered with the minimum number of circles. One method can select the circles to cover the highest number of points in which case the three solid circles 1,2 , and 3 , which respectively cover 12,6 , and 4 points, will be selected. This is not an optimum solution; an optimum solution is to select two dotted circles that cover 11 points each (Dutta, 2009).

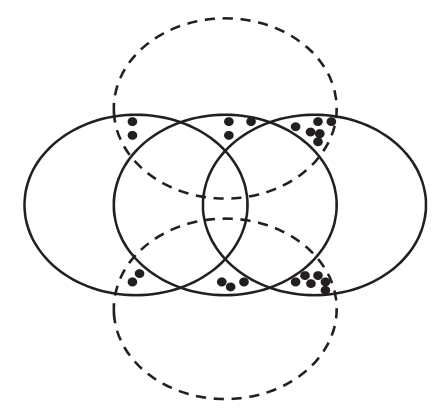

Fig. 1. Geometry of the covering problem sample

In the present research, since proximity to the goods' stock exchange is important too, the general problem pattern (Fig. 2) will be processed through the set covering problem, developed mathematical model, and GA to specify the minimum number and locations of the distribution centers considering the problem inputs (locations of the goods' stock exchange, costs of establishing the centers, and coverage radius).

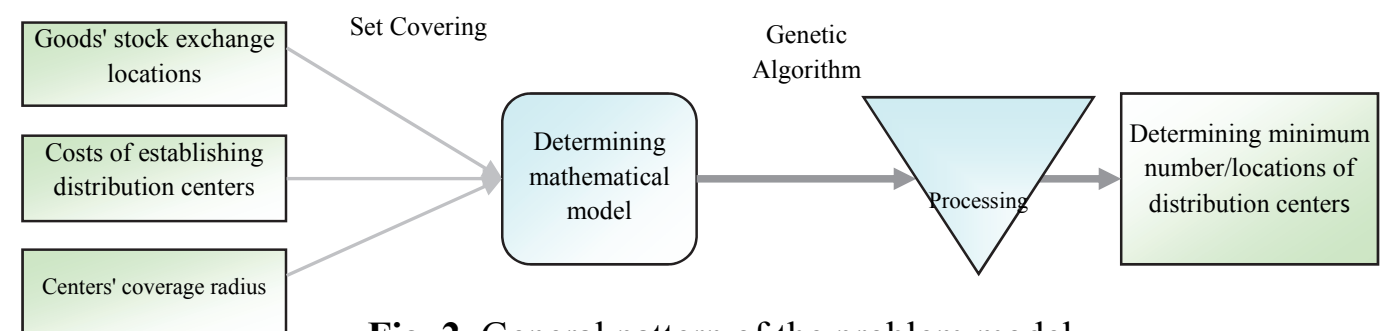

Fig. 2. General pattern of the problem model

As a zero-one programming problem, the set covering is shown as follows:

$$
\text { (P1) } \min Z=\sum_{j=1}^{n} c_{j} x_{j}
$$

where $\mathrm{z}$ is the objective function, $\mathrm{j}$ is the facility (site) index, $c_{j}$ is the cost of allocating a vehicle to site $\mathrm{j}$, and $x_{j}$ is the decision variable.

subject to

$\sum_{j=1}^{n} a_{i j} x_{j} \geq 1, \quad \mathrm{i}=1, \ldots, \mathrm{m}$

$x_{j}=(0,1), \mathrm{j}=1, \ldots, \mathrm{n}$

where $\mathrm{i}$ is demand point (customer) index and $a_{i j}$ are the coverage coefficients.

$x_{j}= \begin{cases}1 & \text { If a vehicle is stationed at site } \mathrm{j} \\ 0 & \text { Otherwise }\end{cases}$
$a_{i j}= \begin{cases}1 & \text { If a vehicle is stationed at site } j \\ 0 & \text { Otherwise }\end{cases}$ 
The problem constraint insures that each of $m$ customers is covered by, at least, one of the $n$ vehicles; the objective is, then, to cover the customers with minimum costs. Eq. (2) insures that each row is covered by at least one column and Eq. (3) shows the absoluteness (obviousness) of the existence of the distribution/service center at the point.

Since most distribution points or goods' stock exchange locations have been developed non-scientifically (traditionally), they do not cover all the customers; therefore, the main objective of the distribution center location is to cover all the customers and, possibly, presence among the rivals and proximity to the goods' stock exchange. Hence, in the mathematical model developed in this research, $d_{j}$ is the distance of facility $\mathrm{j}$ from the goods' stock exchange and other parameters are similar to those of the initial set covering problem model.

$$
\begin{aligned}
& \text { (P2) } \min Z_{1}=\sum_{j=1}^{n} c_{j} x_{j} \\
& \min Z_{2}=\sum_{j=1}^{n} d_{j} x_{j}
\end{aligned}
$$

subject to

$\sum_{j=1}^{n} a_{i j} x_{j} \geq 1, \quad \mathrm{i}=1, \ldots, \mathrm{m}$

$x_{j}=(0,1), \mathrm{j}=1, \ldots, \mathrm{n}$

$x_{j}= \begin{cases}1 & \text { If a vehicle is stationed at site } j \\ 0 & \text { Otherwise }\end{cases}$

$a_{i j}= \begin{cases}1 & \text { If a vehicle is stationed at site } j \\ 0 & \text { Otherwise }\end{cases}$

The set covering problem is a zero-one linear programming model known as an NP-complete optimization problem (as Karp believes) (Sapkota \& Reilly, 2011; Karp, 1972). This problem is discussed when it is necessary that a number of customers exist and each one is served by, at least, one facility with the least possible costs; therefore, the problem investigated in this research will be NP-complete by adding proximity to and the presence in the goods' stock exchange. Since both the minimum costs and proximity are considered, we use $\varepsilon$-constraint method for solution and genetic algorithm is implemented for overcoming the problem's computational complexities and solution of the two-objective function. One of the main objectives of the $\varepsilon$-constraint method is that it is possible to control the number of the produced solutions and intervals by considering the decision makers' criteria; therefore, this method has been used considering the importance of the decision makers' opinion (Khorsi, et al., 2013). The $\varepsilon-$ constraint method is a known applied problem solving method used by many researchers. For instance, Mavrotas (2009) used it to solve multi-objective mathematical programming problems. Berube et al. (2009) used the $\varepsilon$-constraint method to study multi-objective problems and solved them with metaheuristic methods. Amin and Zhong (2013) solved the multi-objective facility location model for a closed-circuit supply chain network under varied conditions. Ramezani et al. (2013) developed a novel multi-objective model for the design of a logistic network and used the $\varepsilon$-constraint method for its solution. Hamedani et al. (2013) developed a multi-objective model for the distribution center location in the supply chain network and solved it by the $\varepsilon$-constraint method. Rath-Gutjahr (2014) used this method to solve location-routing problems. Saffar et al. (2015) proposed a novel multi-objective optimization model for the design of uncapacitated supply chain network and solved it by fuzzy programming and the $\varepsilon$-constraint method. Gutjahr-Dzubur (2016) proposed a two-objective optimization model for distribution centers location with limited inventory at the upper level of which the decision maker selects capacitated distribution center locations with uncovered cost/demand 
objectives and at the lower level the customers select the distribution centers based on proximity and their expected inventory; this model was solved by the $\varepsilon$-constraint and branch-and-bound methods. In the $\varepsilon$-constraint method, the multi-objective optimization problem with the added constraints is changed to a single objective optimization problem. In this method, one of the objective functions is optimized and others are considered as the problem constraints. The related equations are as follows:

$\mathrm{Z}=\min \left(\mathrm{f}_{\mathrm{j}}(x)\right)$

subject to:

$\mathrm{f}_{\mathrm{i}}(\mathrm{x}) \leq \varepsilon_{i} \quad$ for $\mathrm{i}=1,2,3, ., \mathrm{k}$ and $\mathrm{i} \neq \mathrm{j}$

where $\mathrm{j}$ is index of the main objective function for optimization, $\mathrm{i}$ is the index of objective functions considered as constraints, and $\varepsilon$ is the constraint value.

The objective function with the higher priority (cost and number of centers) is considered as the objective function and the other function (proximity) is considered as a constraint. The deformed problem is shown as follows:

(P3) $\min \mathrm{z}=Z_{1}$

subject to

$Z_{2} \leq \varepsilon$

$\sum_{j=1}^{n} a_{i j} x_{j} \geq 1, \quad \mathrm{i}=1, \ldots, \mathrm{m}$

$x_{j}=(0,1), \mathrm{j}=1, \ldots, \mathrm{n}$

$x_{j}= \begin{cases}1 & \text { If a vehicle is stationed at site } j \\ 0 & \text { Otherwise }\end{cases}$

$a_{i j}= \begin{cases}1 & \text { If a vehicle is stationed at site } j \\ 0 & \text { Otherwise }\end{cases}$

where $Z_{1}$ is the main objective function, $Z_{2}$ is the objective function considered as constraint. The $\varepsilon$-value depends on the organization's strategy, and considering the objective (lower costs, higher sales), different numbers are considered. In this research, in every step of algorithm implementation, first each problem was solved with a single objective function of proximity to the goods' stock exchange using MATLAB program and its minimum value was found. Then, this objective function was considered as a constraint and its value was considered with different percents higher than the value found.

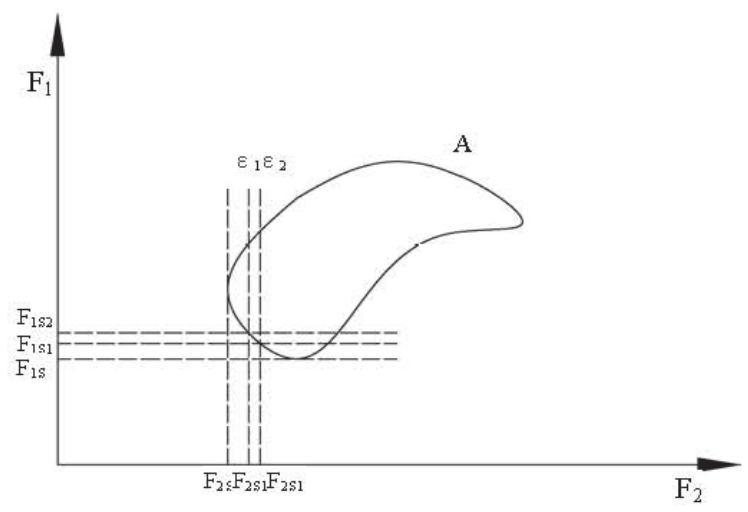

Fig. 3. Geometrical representation of the $\varepsilon$-constraint method 
Fig. 3 shows the procedure of this method for problem A with two objective functions of $F_{1}$ and $F_{2}$. As shown, points $F_{1 s}$ and $F_{2 s}$ show the minimum values of the two functions for a single objective case. It is observed that the solution of the two-objective function will be different with different $\varepsilon_{1}$ values. For instance, for $\varepsilon_{1}$ the values of the first and second objective functions will be respectively $F_{1}=F_{1 s l}$ and $F_{2}=F_{2 s}$.

The step-by-step explanation of the proposed algorithm is as follows:

Step 0: the initial population, number of generations, crossover rate, and mutation rate are determined. It is to be noted that the larger initial populations, the number of generations, and crossover rates can result in an extended search space and, hence, a faster algorithm convergence. Again, since the mutation operator is a marginal one, it is better to assume a lower value for it to prevent a mere random procedure. In this problem, we need to define an $m \times n$ matrix where $m$ is the number of the potential sites. This matrix is binary; its arrays are 1 if a customer is covered by one site and 0 otherwise.

Step 1: the initial population is randomly assumed equal to the number found in Step 0 and the value of the objective function is calculated.

Step 2: mating is done using the uniform crossover operator. Application of a single or two point cutting operator is of course possible in this step, but since the uniform operator is better compared to other methods, it is preferable here. This is how it works: first a $l \times n$ random binary matrix is formed for every pair of chromosomes that are supposed to play the role of parents. According to this matrix, if the number is 1 , the genes of these two chromosomes will not change and if it is 0 , they will be replaced. In the crossover method, two children (offspring) are produced from a pair and the possibility that the parents are transferred to the next generation unaltered is low. Fig. 4 shows an example of such a crossover with $\left[\begin{array}{lllllll}1 & 0 & 1 & 1 & 1 & 0 & 1\end{array}\right]$ positions.

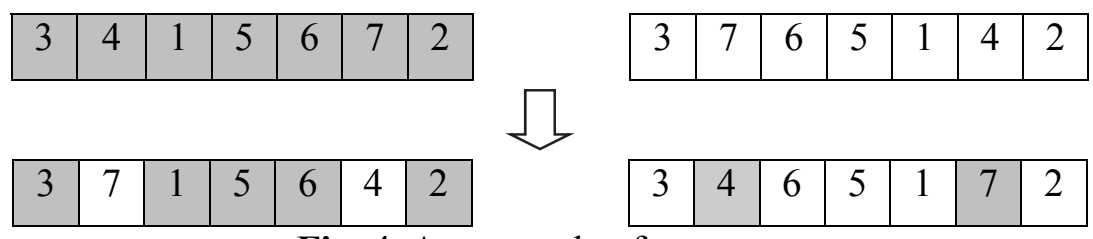

Fig. 4. An example of crossover

To select the parents, we use the ranking method where the population members are sorted (from best to worst) in terms of their fitness value and their best value is selected considering the crossover rate. In this step, some children (offspring) are generated for each of which the objective function is calculated.

Step 3: using the mutation operator, an effort is made to create diversity in the population. This operator randomly selects some chromosomes from the population, cuts each one randomly from a point, and replaces the gene of that point with that of another chromosome. Here again, some children (offspring) are generated for each of which the objective function is calculated. A high mutation rate will result in genetic diversity and dispersion in the population which may delay the convergence. In general, mutation prevents the GA to get trapped in local extermoms. Mutations should not be many because in such cases the GA will turn into a mere random search. Fig. 5 shows an example of mutation with the replacement of the cell 3 gene.

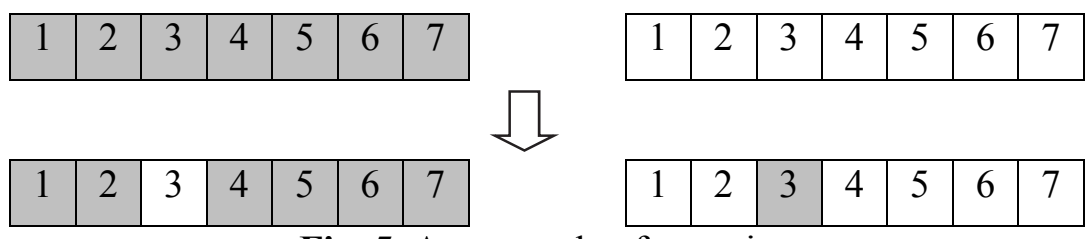

Fig. 5. An example of mutation 
Step 4: selection is performed in this step. All the chromosomes of the previous generation and all the children (offspring) generated from crossover and mutation operators are gathered, sorted based on their fitness values, and a chromosome population (with the required number) is selected from the top of the list. The result of this step is the formation of the population; it is obvious that all the chromosomes in this new population are justified. This way, convergence towards the best solution will be achieved faster and the algorithm implementation time to find an appropriate solution will be shorter. In fact, use is made of the elitism method to maintain a copy of the best chromosomes in the new generation.

Step 5: if the number of iterations (generations) reaches a predetermined value, the algorithm will stop; otherwise, it will return to Step 2.

\section{Evaluation of the algorithm performance}

To evaluate the algorithm performance and make sure of its efficiency, it was necessary to evaluate the solution results of other test problems. In this research, the algorithm was coded with MATLAB (R2013); in the first step, the set covering single objective function (P1) was studied and its MATLAB Code was written. Then, the second objective function was added after good-performance verification.

Step 1: to verify the performance of the single objective MATLAB Code, this research studied 50 set covering-related sample problems taken from the Operation Research Library (Beasley, 1987); details are provided in Table 1.

Table 1

Details of the sample test problems

\begin{tabular}{ccccc}
\hline Problem set & Row $(\mathrm{m})$ & Column $(\mathrm{n})$ & Density $(\%)$ & No of problems \\
\hline 4 & 200 & 1000 & 2 & 10 \\
5 & 200 & 2000 & 2 & 10 \\
6 & 200 & 1000 & 5 & 5 \\
A & 300 & 3000 & 2 & 5 \\
B & 300 & 3000 & 5 & 5 \\
C & 400 & 4000 & 2 & 5 \\
D & 400 & 4000 & 5 & 5 \\
E & 50 & 500 & 20 & 5 \\
\hline
\end{tabular}

where $m$ is the number of customers (facility takers), $n$ is the number of distribution centers (facility providers), density means the number of $1 \mathrm{~s}$ in the covering matrix, and the last column shows the number of problems in each set. For instance, the first row in the table means problem set 4 contains covering matrixes of $200 \times 1000$ with $2 \%$ density and involves (according to the reference mentioned) 10 problems (scp $4.1 \ldots$ scp 4.10). In this problem, there are 1000 potential customer facility providing locations through which 200 points should be covered. In each case, the algorithm has been run 10 times with mutation and crossover rates of respectively 0.1 and 0.8 and the results have verified the proper performance of the MATLAB Code.

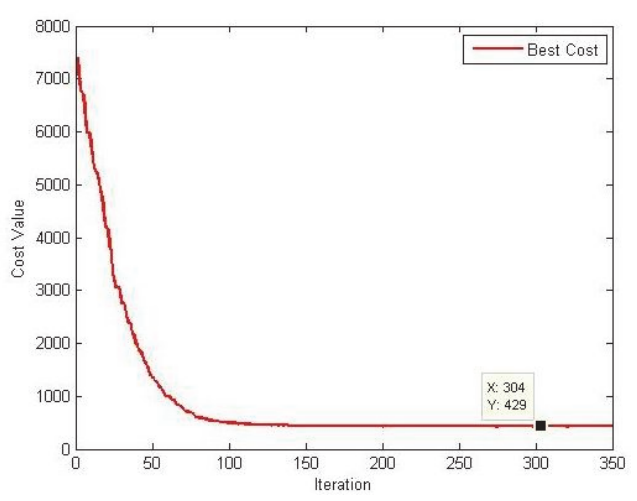

Fig. 6. Solution of covering problem scp 4.1

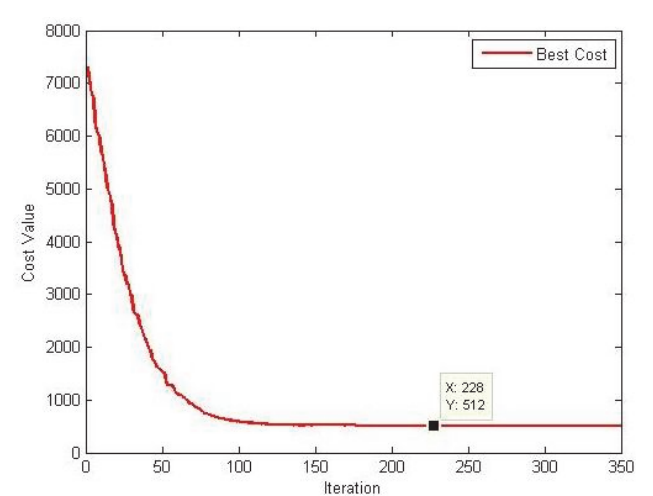

Fig. 7. Solution of covering problem scp 4.2 
For sample, Figs. 6 and 7 show the graphs for problems scp 4.1 and scp 4.2; as shown, the distribution centers' establishment costs are high in the initial iterations. Then, with some improvements in the next iterations, they gradually decrease and finally reach a constant value which shows the optimum solution and its convergence. For example, the point specified on Fig. 6 shows the function's objective (sum of the distribution/service centers' establishment costs) in iteration 304 equals 429 considering the coverage of all the desired points; this cost, which is shown in iteration 228 in Fig. 7, is 512.

Step 2: By considering the good performance in Step 1, the second objective function (proximity to stock exchange) is added to the initial MATLAB Code and solved with the $\varepsilon$-constraint method. To solve the new problem, the distances of the distribution centers from the stock exchange are found and the second objective function is calculated considering a specified $\varepsilon$-value. If the center is out of range, it will be omitted by adding a large number to the objective function fitness. Finally, the centers with best fitness values are specified and the facility giver to each customer is determined.

Table 2

Results of the two-objective function

\begin{tabular}{|c|c|c|c|c|c|}
\hline Problem & Row m & Column n & Density $\%$ & Single-objective optimum value & two-objective optimum value \\
\hline 4.1 & 200 & 1000 & 2 & 429 & 753 \\
\hline 4.2 & 200 & 1000 & 2 & 512 & 866 \\
\hline 4.3 & 200 & 1000 & 2 & 516 & 824 \\
\hline 4.4 & 200 & 1000 & 2 & 494 & 792 \\
\hline 4.5 & 200 & 1000 & 2 & 515 & 820 \\
\hline 4.6 & 200 & 1000 & 2 & 560 & 872 \\
\hline 4.7 & 200 & 1000 & 2 & 430 & 691 \\
\hline 4.8 & 200 & 1000 & 2 & 510 & 783 \\
\hline 4.9 & 200 & 1000 & 2 & 641 & 1011 \\
\hline 4.10 & 200 & 1000 & 2 & 521 & 822 \\
\hline 5.1 & 200 & 2000 & 2 & 253 & 401 \\
\hline 5.2 & 200 & 2000 & 2 & 302 & 494 \\
\hline 5.3 & 200 & 2000 & 2 & 232 & 360 \\
\hline 5.4 & 200 & 2000 & 2 & 248 & 384 \\
\hline 5.5 & 200 & 2000 & 2 & 211 & 338 \\
\hline 5.6 & 200 & 2000 & 2 & 213 & 339 \\
\hline 5.7 & 200 & 2000 & 2 & 293 & 465 \\
\hline 5.8 & 200 & 2000 & 2 & 288 & 460 \\
\hline 5.9 & 200 & 2000 & 2 & 285 & 441 \\
\hline 5.10 & 200 & 2000 & 2 & 265 & 424 \\
\hline 6.1 & 200 & 1000 & 5 & 138 & 212 \\
\hline 6.2 & 200 & 1000 & 5 & 146 & 233 \\
\hline 6.3 & 200 & 1000 & 5 & 145 & 232 \\
\hline 6.4 & 200 & 1000 & 5 & 131 & 204 \\
\hline 6.5 & 200 & 1000 & 5 & 161 & 249 \\
\hline A. 1 & 300 & 3000 & 2 & 253 & 401 \\
\hline A. 2 & 300 & 3000 & 2 & 259 & 399 \\
\hline A. 3 & 300 & 3000 & 2 & 237 & 362 \\
\hline A. 4 & 300 & 3000 & 2 & 234 & 365 \\
\hline A. 5 & 300 & 3000 & 2 & 236 & 369 \\
\hline B. 1 & 300 & 3000 & 5 & 71 & 102 \\
\hline B. 2 & 300 & 3000 & 5 & 76 & 110 \\
\hline B. 3 & 300 & 3000 & 5 & 80 & 113 \\
\hline B. 4 & 300 & 3000 & 5 & 79 & 112 \\
\hline B. 5 & 300 & 3000 & 5 & 75 & 108 \\
\hline C. 1 & 400 & 4000 & 2 & 230 & 362 \\
\hline C. 2 & 400 & 4000 & 2 & 219 & 349 \\
\hline C. 3 & 400 & 4000 & 2 & 245 & 385 \\
\hline C. 4 & 400 & 4000 & 2 & 219 & 349 \\
\hline C. 5 & 400 & 4000 & 2 & 215 & 341 \\
\hline D. 1 & 400 & 4000 & 5 & 60 & 80 \\
\hline D. 2 & 400 & 4000 & 5 & 66 & 86 \\
\hline D. 3 & 400 & 4000 & 5 & 73 & 108 \\
\hline D. 4 & 400 & 4000 & 5 & 62 & 83 \\
\hline D. 5 & 400 & 4000 & 5 & 61 & 81 \\
\hline E.1 & 50 & 500 & 20 & 5 & 11 \\
\hline E. 2 & 50 & 500 & 20 & 5 & 11 \\
\hline E. 3 & 50 & 500 & 20 & 5 & 11 \\
\hline E. 4 & 50 & 500 & 20 & 5 & 11 \\
\hline E. 5 & 50 & 500 & 20 & 5 & 11 \\
\hline
\end{tabular}

In evolutionary algorithms, since the initial population is selected randomly, results will be different with different implementations; therefore, it is necessary to run the algorithm many times but with similar parameters to reveal the differences in the results. In this research, the algorithm was run 10 times and 
the optimum solutions for 50 sample problems with $\varepsilon$-values of about $25 \%$ higher than the minimum value of the single objective of proximity to the stock exchange are presented in Table 2. Figs. 8 and 9 show the graphs of problems scp 4.1 and scp 4.2 with the second objective function added.
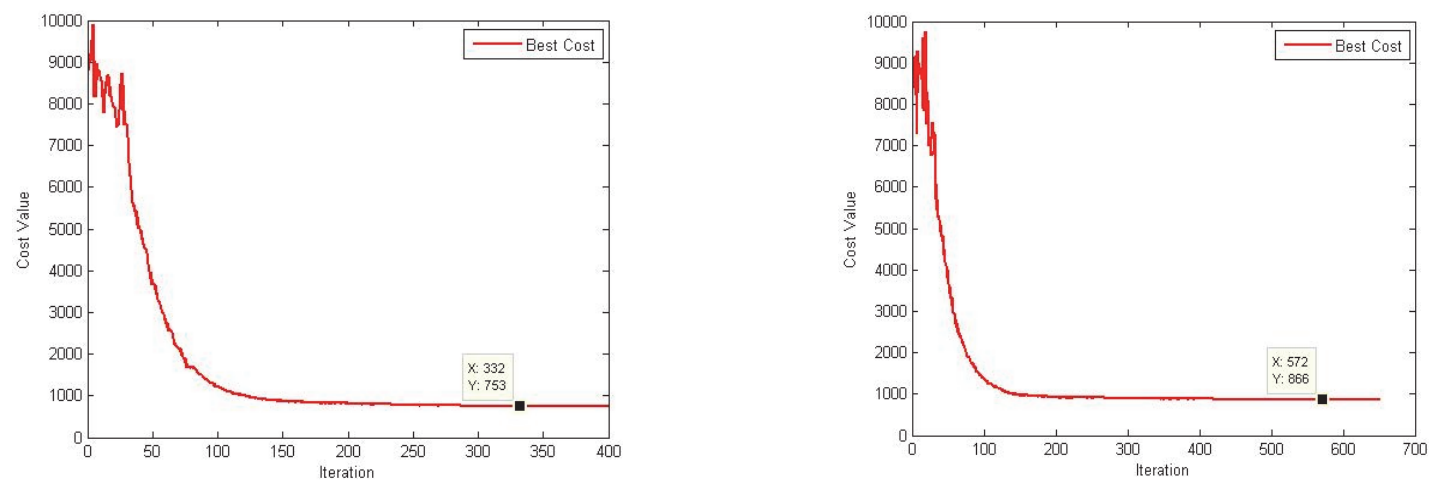

Fig. 8. Solution of two-objective problem scp 4.1 Fig. 9. Solution of two-objective problem scp 4.2

In the initial iterations, since the $\varepsilon$-constraint has been considered and the value of the second objective function is higher than the constraint value, the first objective function value will have non-uniform variations, but in the next iterations, since the second objective function value becomes less than the constraint value, the distribution centers' establishment costs gradually decrease and finally reach a constant value which shows the problem optimum solution and its convergence. The point specified on the first graph shows the function's objective (total distribution/service centers' costs) in iteration 322 equals 753 considering the coverage of all the desired points. In the second graph too, these costs equal 866 in iteration 572. For more detailed studies, Fig. 10 shows the solutions of the two single objective functions independently. The value of the first objective function (establishment costs of covering customers) equals 429 (in iteration 301) with the corresponding distance of 10333. The value of the second objective function (distance from the stock exchange) at this point equals 1599 with the corresponding establishment costs of 3658 .

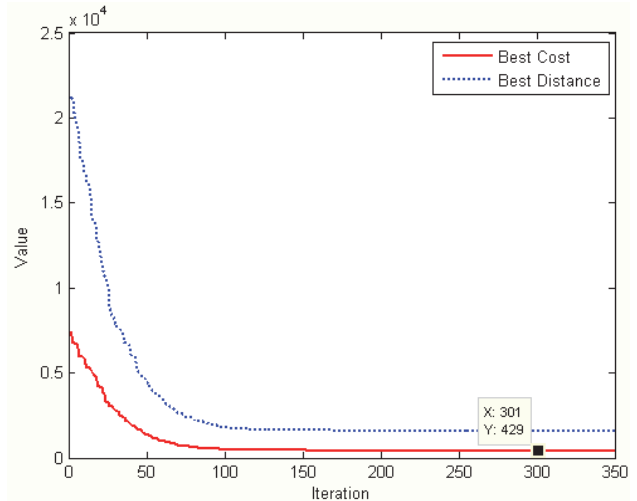

Fig. 10. Problem solution (two independent objective functions)

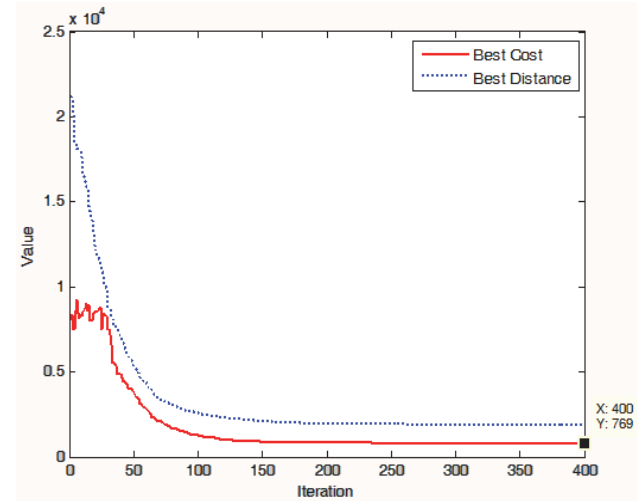

Fig. 11. Problem solution (two-objective function)

Fig. 11 shows the two-objective function solution (considering a constraint value of about $10 \%$ higher than the minimum value of the single-objective function). The optimum value of the first objective function (establishment costs) equals 769 with the corresponding distance of 774, and the optimum value of the second objective function (distance from the stock exchange) is 1859 with the corresponding establishment costs of 4348 . The model has been solved with different constraint values and results are shown in Table 3. 
Table 3

Constraint-based solution results of the two-objective function

\begin{tabular}{ccccc}
\hline Constraint increase & \multicolumn{2}{c}{ First objective function } & \multicolumn{2}{c}{ Second objective function } \\
\cline { 2 - 5 }$(\%)$ & Cost $(\mathrm{C})$ & Corresponding distance (d) & Distance (D) & Corresponding costs (c) \\
\hline 10 & 769 & 7747 & 1859 & 4348 \\
20 & 755 & 7584 & 1639 & 3367 \\
30 & 741 & 7434 & 1444 & 2640 \\
40 & 726 & 7293 & 1272 & 2070 \\
50 & 712 & 7161 & 1134 & 1645 \\
\hline
\end{tabular}

With an increase in $\varepsilon$, the optimum value of the two-objective problem will get close to that of the singleobjective problem, and with a decrease in $\varepsilon$, the optimum value will increase. The program implementation with different $\varepsilon$-values shows that, on average, a $10 \%$ increase in the $\varepsilon$-value will cause a $2 \%$ decrease in costs meaning that when the effect of the second objective function is reduced, the value of the first (cost) decreases and gets close to the single-objective case. Fig. 12 shows the set of Pareto optimal solutions considering $\varepsilon$-values $10 \%$ higher than the minimum value of the second objective function. As shown, getting close to the Origin means more desirable solutions.

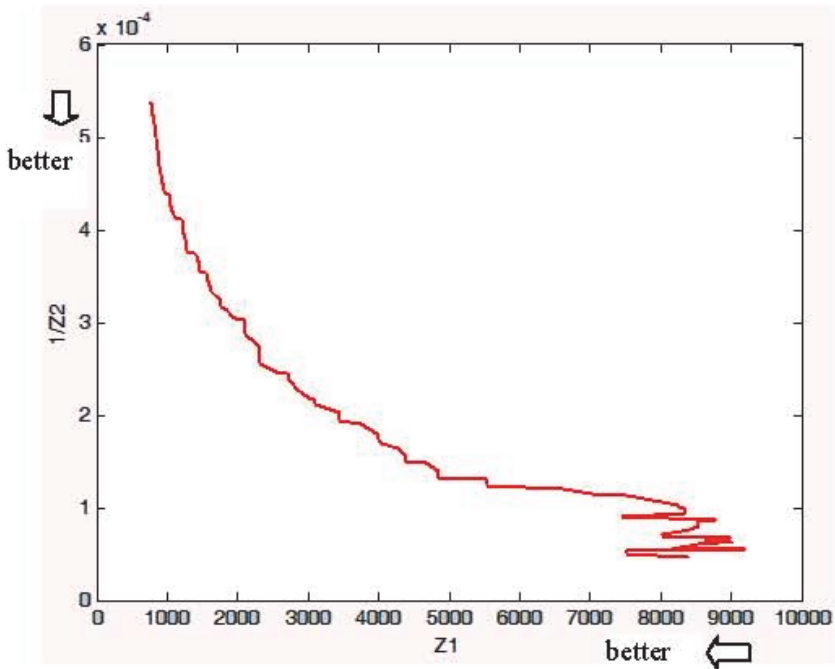

Fig. 12. Set of Pareto optimal solutions (x-axis $Z 1$ : solutions of the first objective function; $y$-axis $1 / Z 2$ : inverse of the solutions of the second objective function)

In the set of Pareto optimal solutions, one solution cannot be preferred to the other in a pair because a decrease in the optimum point of the first objective function will cause an increase in that of the second and vice versa; therefore, it is possible to achieve a desirable point based on the company's strategy.

\section{Summary and conclusions}

Facility location plays an important part in heavy and long-term investments because an appropriate location attracts many customers and if at the beginning a center is established at its proper place, its related business will succeed and highly benefit its investor. This research involves two objectives one of which is the minimum establishment costs considering customers covering and the other is proximity to the stock exchange. The proposed model uses the $\varepsilon$-constraint method, adds the second objective function as another constraint, and finds the optimum solution through the metaheuristic GA. Results have shown that the final selection of the distribution centers could depend on the company's strategy. For instance, although it is possible to establish a distribution center in the stock exchange, the company may not minimize the costs. A very important achievement in this research is developing the set covering model to one with two objectives meaning that while the establishment costs of the distribution/service 
centers are minimized, their distances to the stock exchange are also minimized. Implementing the model with different $\varepsilon$-values shows that for a $10 \%$ increase in the $\varepsilon$-value (that is reducing the number of distribution centers and increasing the distance to reach them) there will be about $2 \%$ decrease in the establishment costs considering the customers' set covering.

Lack of a meaningful difference in different consecutive algorithm iterations is an indication of its robustness; the algorithm converges and yields acceptable results for different problems (with an initial population and iterations specific to each problem). Better solutions are possible with higher initial populations (spending more time), but if the decision making time is limited a low initial population will also yield an acceptable result. The minimum value of the objective function will reduce with an increase in the number of generations.

\section{Acknowledgement}

The author would like to thank the anonymous referees for constructive comments on earlier version of this paper.

\section{References}

Aickelin, U. (2002). An indirect genetic algorithm for set covering problems. Journal of the Operational Research Society, 1118-1126.

Akgun, I., Gumuşbuga, F., \& Tansel, B. (2015). Risk based facility location by using fault tree analysis in disaster management. Omega, 52, 168-179.

Amin, S. H., \& Zhang, G. (2013). A multi-objective facility location model for closed-loop supply chain network under uncertain demand and return. Applied Mathematical Modelling, 37(6), 4165-4176.

Anderssen, T. W., \& Lindestad, B. (1998). Customer Loyalty and Complex Service. International Journal of Service Industry Management, 9, 7-32.

Ardjmand, E., Weckman, G., Park, N., Taherkhani, P., \& Singh, M. (2015). Applying genetic algorithm to a new location and routing model of hazardous materials. International Journal of Production Research, 53(3), 916-928.

Ardjmand, E., Young, W. A., Weckman, G. R., Bajgiran, O. S., Aminipour, B., \& Park, N. (2016). Applying genetic algorithm to a new bi-objective stochastic model for transportation, location, and allocation of hazardous materials. Expert Systems with Applications, 51, 49-58.

Berube, J. F., Gendreau, M., \& Potvin, J. Y. (2009). An exact $\epsilon$-constraint method for bi-objective combinatorial optimization problems: Application to the Traveling Salesman Problem with Profits. European Journal of Operational Research, 194(1), 39-50.

Brandeau, M. L., \& Chiu, S. S. (1989). An overview of representative problems in location research. Management science, 35(6), 645-674.

Chiu, S. I., Cheng, C. C., Yen, T. M., \& Hu, H. Y. (2011). Preliminary research on customer satisfaction models in Taiwan: A case study from the automobile industry. Expert Systems with Applications, 38(8), 9780-9787.

Dell'Olmo, P., Ricciardi, N., \& Sgalambro, A. (2014). A Multiperiod Maximal Covering Location Model for the optimal location of intersection safety cameras on an urban traffic network. Procedia-Social and Behavioral Sciences, 108, 106-117.

Dinler, D., Tural, M. K., \& Iyigun, C. (2015). Heuristics for a continuous multi-facility location problem with demand regions. Computers \& Operations Research, 62, 237-256.

Dutta, H. S. (2009). Survey of approximation algorithms for set cover problem. University of North Texas.

Farahani, R. Z., \& Asgari, N. (2007). Combination of MCDM and covering techniques in a hierarchical model for facility location: A case study. European Journal of Operational Research, 176(3), 18391858. 
Farahani, R. Z., Hassani, A., Mousavi, S. M., \& Baygi, M. B. (2014). A hybrid artificial bee colony for disruption in a hierarchical maximal covering location problem. Computers \& Industrial Engineering, $75,129-141$.

Gutjahr, W. J., \& Dzubur, N. (2016). Bi-objective bilevel optimization of distribution center locations considering user equilibria. Transportation Research Part E: Logistics and Transportation Review, $85,1-22$.

Haimes, Y. Y., Ladson, L. S., \& Wismer, D. A. (1971). Bicriterion formulation of problems of integrated system identification and system optimization. IEEE Transactions on Systems Man and Cybernetics, (3), 296.

Hamedani, S., Jabalameli, M., \& Bozorgi-Amiri, A. (2013). A multi-objective model for locating distribution centers in a supply chain network considering risk and inventory decisions. Management Science Letters, 3(4), 1077-1088.

Hosage, C. M., \& Goodchild, M. F. (1986). Discrete space location-allocation solutions from genetic algorithms. Annals of Operations Research, 6(2), 35-46.

Hosseininezhad, S. J., Jabalameli, M. S., \& Naini, S. G. J. (2013). A continuous covering location model with risk consideration. Applied Mathematical Modelling, 37(23), 9665-9676.

Jaramillo, J. H., Bhadury, J., \& Batta, R. (2002). On the use of genetic algorithms to solve location problems. Computers \& Operations Research, 29(6), 761-779.

Karp, R. M. (1972). Reducibility among combinatorial problems (pp. 85-103). springer US.

Klose, A., \& Drexl, A. (2005). Facility location models for distribution system design. European Journal of Operational Research, 162(1), 4-29.

Khorsi, M., Bozorgi-Amiri, A., \& Ashjari, B. (2013). A nonlinear dynamic logistics model for disaster response under uncertainty. Significance, 3, 4.

Malekinezhad, A., Shirazi, E., \& Aryanezhad, M. (2011). A multi-objective set covering problem: A case study of warehouse allocation in truck industry. Management Science Letters, 1(1), 73-80.

Mavrotas, G. (2009). Effective implementation of the $\varepsilon$-constraint method in multi-objective mathematical programming problems. Applied mathematics and computation, 213(2), 455-465.

Ramezani, M., Bashiri, M., \& Tavakkoli-Moghaddam, R. (2013). A new multi-objective stochastic model for a forward/reverse logistic network design with responsiveness and quality level. Applied Mathematical Modelling, 37(1), 328-344.

Rath, S., \& Gutjahr, W. J. (2014). A math-heuristic for the warehouse location-routing problem in disaster relief. Computers \& Operations Research, 42, 25-39.

Ren, Y., \& Awasthi, A. (2015). Investigating metaheuristics applications for capacitated location allocation problem on logistics networks. In Chaos Modeling and Control Systems Design (pp. 213238). Springer International Publishing.

Saffar, M. H. S. G., \& Razmi, J. (2015). A new multi objective optimization model for designing a green supply chain network under uncertainty. International Journal of Industrial Engineering Computations, 6(1), 15-32.

Santouridis, I., \& Trivellas, P. (2010). Investigating the impact of service quality and customer satisfaction on customer loyalty in mobile telephony in Greece. The TQM Journal, 22(3), 330-343.

Sapkota, N., \& Reilly, C. H. (2011). Simulating realistic set covering problems with known optimal solutions. Computers \& Industrial Engineering, 61(1), 39-47

Schmid, V., \& Doerner, K. F. (2010). Ambulance location and relocation problems with time-dependent travel times. European Journal of Operational Research, 207(3), 1293-1303.

Tang, S. H., Boyer, O., Pedram, A., Mohd Yusuff, R., \& Zulkifli, N. (2013). A review on multiple criteria undesirable facility location problems. Journal of Basic and Applied Scientific Research, 3(8), 708713. 
Wang, K. J., Makond, B., \& Liu, S. Y. (2011). Location and allocation decisions in a two-echelon supply chain with stochastic demand-A genetic-algorithm based solution. Expert Systems with Applications, 38(5), 6125-6131.

Wang, B., Fu, X., Chen, T., \& Zhou, G. (2014). Modeling Supply Chain Facility Location Problem and Its Solution Using a Genetic Algorithm. Journal of Software, 9(9), 2335-2341.

Ye, L., Ye, C., \& Chuang, Y. F. (2011). Location set covering for waste resource recycling centers in Taiwan. Resources, Conservation and Recycling, 55(11), 979-985. 